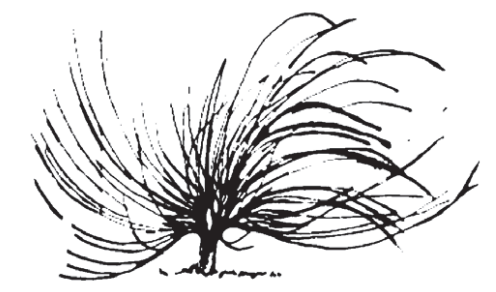

\title{
Sensibilidad, Afectividad y Reflexión en las Aulas: Una Carencia en la Labor Docente
}

\author{
Marco Madriz Rojas ${ }^{1}$ \\ Universidad Nacional \\ Heredia, Costa Rica \\ marcvs87@gmail.com \\ Stephanie Navarro Castro \\ Universidad Nacional \\ Heredia, Costa Rica \\ stephanie2101@hotmail.es
}

\begin{abstract}
Resumen
La sensibilidad debe ser una cualidad propia de la labor docente para lograr una mejor interacción con sus estudiantes, reconociéndolos como personas con derecho. No obstante, en el Sistema Educativo Nacional, prevalecen discursos de acercamiento hacia los estudiantes que pocas veces son llevados a la práctica, dejando de lado la proximidad afectiva y creando barreras que separan en gran medida a los educadores de sus estudiantes. En este sentido, es necesaria la promoción de estrategias educativas integrales desde la reflexión del docente que devuelvan la palabra a los y las estudiantes y reconozcan su identidad individual, de modo que la educación sea una herramienta para alcanzar la realización personal de los educandos.
\end{abstract}

Recibido: 28 de noviembre de 2013 - Aprobado: 12 de febrero de 2014

1 Estudiante de Licenciatura en la Enseñanza de los Estudios Sociales y Educación Cívica, Universidad Nacional.

2 Estudiante de Licenciatura en la Enseñanza de los Estudios Sociales y Educación Cívica, Universidad Nacional. 
Palabras Claves: sensibilidad, afectividad, reflexión, ambiente de aula

\begin{abstract}
Sensitivity should be an intrinsic quality of teaching to achieve better interaction with students, recognizing them as persons. However, in our national education system, discourses aiming to approach students prevail, but they are rarely put into practice, leaving aside the emotional closeness and creating barriers that separate educators from their students to a great extent. In this regard, the promotion of comprehensive educational strategies is necessary from the teacher's reflection to return a voice to the students and recognize their individual identity, so that education becomes a tool to achieve the personal development of learners.
\end{abstract}

Keywords: sensitivity, affectivity, reflection, classroom environment

\title{
Aspectos introductorios
}

L os y las docentes, si bien es cierto, no son los actores y actrices principales del proceso de enseñanza y aprendizaje, sino que constituyen sólo una parte del proceso educativo. No obstante, la labor que llevan a cabo educadores y educadoras es determinante, a la hora de establecer ambientes de aula placenteros para los y las estudiantes. En este sentido, cuando la autoridad de la figura docente se convierte en autoritarismo, crea una barrera, reduciendo el grado de interacción con los y las estudiantes, lo que incide en la pérdida de comunicación y, con ello, en la reducción del proceso educativo a una relación de dominio.

Al mismo tiempo, la potestad atribuida al cuerpo docente por la naturaleza de su profesión, exige la puesta en práctica de actitudes humanistas que acorten las distancias con los y las estudiantes y reflejen su sensibilidad en torno a sus realidades, de modo que no se visualicen como entes aislados sino que, reflexionando acerca de los elementos que envuelven su contexto de aula, puedan crear las condiciones para la mejora de la práctica educativa. Los y las docentes -como mediadores del proceso de enseñanza y aprendizaje - se convierten, a la postre, en 
objeto de estudio de sus propios estudiantes, quienes de formas diversas llegan a catalogar a sus y profesoras de acuerdo con la actitud que reflejen en la labor de aula. Por lo tanto, dentro del trabajo docente no está de más el reflejo de su carisma, respeto y principios éticos, sobre todo, al tomar en cuenta cómo muchas veces los mismos son considerados modelos a seguir.

\section{El quehacer docente en torno a la sensibilidad, afectividad y reflexión}

La práctica educativa, por su naturaleza, constituye una interrelación entre personas con igualdad en dignidad y derechos. El trabajo de los y las educadores(as) debe alejarse de la idealización de una función operaria, caracterizada por la manipulación de máquinas para la obtención de bienes; la educación enfatiza más bien en la formación de personas, seres humanos dotados de sentimientos. Esto exige que las y los formadores(as) reconozcan la importancia de la sensibilidad en su trato con los y las estudiantes. Esta sensibilidad tiene como base su reconocimiento como personas, con derecho a la dignidad, a la aceptación de su identidad y al respeto mutuo, lo que conlleva a una equiparación, donde el docente no se posicione por encima de sus estudiantes, sino que los visualice como iguales para fortalecer la unión de grupo a partir de la consolidación de lazos de proximidad afectiva. Paulo Freire (2008, pp. 131-132) se refiere a la necesidad de expresar la afectividad en el proceso educativo en estos términos:

Es necesario estar abierto al gusto de querer bien y, a veces, al desafío de querer bien a los educandos y a la propia práctica educativa de la cual participo. Esta apertura al querer bien (...) significa, de hecho, que la afectividad no me asusta, que no tengo miedo de expresarla. Esta apertura al querer bien significa, la manera que tengo de sellar automáticamente mi compromiso con los educandos, en una práctica específica del ser humano.

La afectividad, por lo tanto, es inherente a la labor docente, pues facilita posibilidades de relación, donde docente y estudiante puedan interactuar en ambientes armónicos, erradicando la figura del educador lejano, opresor e insensible ante la presencia de sus estudiantes. Eso sí, se debe tener claro que esta afectividad no debe interferir en el ejercicio 
de la ética docente, ya que este grado de cercanía no debe causar parcialidad, priorizando a unos sobre otros, sino que debe trasmitirse de forma integral, igualitaria, teniendo claro que no se trata de una relación familiar sino profesional.

El desarrollo de lazos de sensibilidad y afectividad, permitirá a los y las docentes llevar a cabo una práctica reflexiva a través de la cual se resalte el papel del estudiante y se creen las condiciones necesarias para la atención de sus necesidades no sólo educativas sino también emocionales. Esta reflexión debe considerar en un primer momento el contexto al que pertenecen los y las estudiantes; no es posible que las y los educadores(as), se sientan ajenos a las comunidades en las que ejercen su práctica educativa, aún si no pertenecen a las localidades en las que laboran, deben estar inmersos de su realidad social, cultural y económica, pues de otro modo no existirá una concientización respecto a su entorno educativo. Igualmente, la reflexión respecto al contexto conlleva a la función del docente como un agente activo en su comunidad de trabajo, involucrándose en la resolución de sus problemáticas y en las vivencias sociales, así como creando espacios en el aula para la sensibilización acerca de las temáticas de interés comunal y sus posibles soluciones.

\section{Una mirada hacia el sistema educativo costarricense: ¿Se promue- ve la sensibilidad, afectividad y reflexión?}

Como queda dicho, la sensibilidad, afectividad y reflexión forman parte indispensable dentro de la labor docente y de la motivación hacia los y las estudiantes. Al respecto, el Consejo Superior de Educación de Costa Rica (2008, p. 9), ha enfatizado en las características que debe tener la labor docente, estableciendo, en un primer momento, la necesidad de poner en práctica la sensibilización como una forma de orientar hacia la criticidad entre las y los educandos, siendo esta una cualidad innata en los seres humanos. Al mismo tiempo, esta instancia considera que la política educativa nacional debe basarse en principios humanistas, racionalistas y constructivistas con el fin de reconocer la dignidad y el progreso, incentivando la realización plena de los y las estudiantes como prioridad dentro del sistema educativo.

A pesar de los postulados proclamados por las instancias educativas, es de reconocer que la realidad en las aulas deja mucho que desear respecto a su cumplimiento. En la actualidad, persiste todavía 
un divorcio entre el currículum pre-escrito y el desarrollado. De forma contradictoria a los principios humanistas propuestos, el Sistema Educativo costarricense está diseñado para el acatamiento de un programa de estudios específico cargado de múltiples contenidos, que debe ser abordado en su totalidad en un periodo determinado. Este sistema incide en que no pocos docentes se excusen en la falta de tiempo, considerándola como una limitante para establecer relaciones estrechas con sus estudiantes y conocer la realidad de los contextos en que se desenvuelven. De este modo, una parte del cuerpo docente se limita a un cumplimiento superficial de su labor, dedicándose simplemente a impartir contenidos, considerando la "trasmisión de conocimientos" como el eje fundamental de su ejercicio profesional, otorgando a los Programas de Estudio el papel central del proceso de aprendizaje y dejando a los y las estudiantes en una función pasiva. Con ello, el proceso de enseñanza y aprendizaje se aleja de esos principios humanistas y se crea un distanciamiento entre los y las educadoras y los contextos en que se desenvuelven, lo que impide una verdadera reflexión para la atención de las diferentes necesidades que se presentan en las aulas.

Una carencia más presente en la realidad nacional, la constituye el desconocimiento que existe desde los docentes hacia sus estudiantes y la reflexión que pueda surgir a partir de ello, lo cual muchas veces ha sido atribuido asimismo, a la necesidad de cumplir con el currículo de estudio. En este sentido, prevalecen los educadores que no se dan a la tarea siquiera de conocer aspectos básicos de los y las estudiantes; no saben sus nombres, no se interesan por sus vivencias, se afanan en homogenizar a un grupo integrado por personas muy diferentes, cada una con características muy particulares. Los pesados escritorios fungen como un muro que separa al docente de los estudiantes. El profesional en educación permanece inmerso en su mundo, como el poseedor del conocimiento, alejado de los "seres no pensantes", receptores limitados. Al reproducirse de forma constante estos patrones que ponen por encima la figura docente, los y las estudiantes terminarán asumiendo esta idealización como válida, considerándose incapaces, lo que verá limitadas sus proyecciones de realización personal a futuro, al tiempo que queda de lado el fortalecimiento de su autoestima y la construcción de su identidad.

El Consejo Superior de Educación ha insistido también en la existencia de una coherencia entre la teoría y la práctica como forma de propiciar la reflexión y sensibilizar a los estudiantes sobre sus 
contextos, sin embargo, la realidad con respecto a esto es contraria a su cumplimiento, pues sigue imperando en gran medida el tradicionalismo y las clases magistrales que siguen restando peso a la palabra del estudiante. La implementación de estrategias que promuevan la sensibilización y afectividad será fundamental para el reconocimiento de los y las estudiantes como personas y otorgará legitimidad acerca de las diferentes formas de pensar sobre su realidad. Así lo hace notar María Cecilia Garcezal al establecer que "a través de la vivencia de situaciones de solidaridad y de la creación de espacios de relación y reflexión a partir de las experiencias de grupo, se crean las condiciones de participación crítica en la realidad en que viven" (Consejo Superior de Educación, 2008, p. 103).

La educación, entonces, sigue estando permeada de relaciones de dominio, donde se da prioridad a una figura docente protagonista, en detrimento de la atención de las diversas necesidades que presentan las y los estudiantes. Esta relación responde también a la jerarquización establecida en el sistema educativo, donde la organización reproduce sistemas de poder, estando el docente por debajo del cuerpo administrativo de las instituciones educativas, pero por encima de los estudiantes que tiene a su cargo, por ser el adulto la figura de autoridad frente a los jóvenes.

Las exclusiones de diferente tipo siguen estando presentes en el actuar del cuerpo docente. Si bien es cierto, que en materia educativa se han dado avances que pretenden incentivar la integración en términos culturales, otras prácticas mantienen todavía latentes patrones de exclusión a nivel de aula. Estas prácticas se dan en gran medida por la pérdida de lazos de afectividad desde el docente hacia el estudiante, quien no representa para él un sujeto de interés. Así por ejemplo, tendencias como el favoritismo hacia ciertos estudiantes crean rupturas a nivel grupal, lo que puede afectar el estado emocional de los educandos. Estas tendencias, asimismo, se encargan de invisibilizar a aquellos y aquellas estudiantes más lejanos, los que muestran una personalidad pasiva a diferencia de compañeros(as) más activos(as). Sin embargo, son precisamente estos estudiantes quienes necesitan un acercamiento cálido por parte del docente, que les motive y les incite a no ser actores y actrices recluidos, impulsando su inclusión y participación desde estrategias integrales que les conceda protagonismo en la dinámica de clase. Ante estas condicionantes, es necesario que el docente realice una relectura de grupo, donde priorice las realidades de los y las estudiantes 
para que, a partir de lo mismo, pueda satisfacer tomar las medidas para sus necesidades de acuerdo con sus cualidades, vivencias y anhelos.

Naturalmente, la sensibilidad, afectividad y reflexión son rasgos propios no sólo de la naturaleza humana, sino que, de modo particular, corresponden en gran medida a la vocación docente. A pesar de ello, prevalece una actitud de condicionamiento para la práctica de estas acciones frente a la satisfacción de intereses económicos. A este respecto, los y las docentes ponen límites a su acercamiento con los y las estudiantes y dejan de lado su compromiso bajo la premisa de condiciones laborales insuficientes, aduciendo en algunos casos factores como remuneraciones económicas exiguas y exceso de carga laboral. Bajo estas condiciones se engrosan las barreras que distancian a los y las estudiantes de sus docentes, donde estos últimos llegan a ser visualizados como entes extraños a su realidad, que no pertenecen a su entorno y se restringen a cumplir a diario con un protocolo estructurado que consiste en entrar a la institución educativa, impartir contenido y marcharse sin más. Esto se ve reflejado igualmente cuando se deja de lado la cortesía en la interacción docente-estudiante, donde no existen saludos ni despedidas, tampoco palabras de motivación ni interés por relacionarse de forma estrecha con sus contextos cercanos. En este sentido, pareciera ser que los docentes de forma lamentable obvian la existencia de sus estudiantes, lo que se evidencia a la hora de planificar las clases de una forma estandarizada, como una especie de ritual establecido para ser implementado en sujetos de paso, que van y vienen una y otra vez, y cuya historia particular no tiene importancia.

Así pues, el diseño del sistema educativo nacional, ha sido permisivo de una labor docente conformista y poco comprometida. El trabajo simplista y mediocre incide, a la postre, en dejar de lado la tarea reflexiva docente. En panoramas como éste, no se da cabida a la sensibilidad como parte del quehacer docente, al considerársele como una cualidad externa al proceso de enseñanza-aprendizaje, donde se supone que debe primar una relación impersonal y carente de afecto.

\section{La promoción de la sensibilidad, afectividad y reflexión en las au- las: Lo que el estudiante necesita}

Como respuesta a los efectos adversos de las prácticas educativas insensibles y mecanicistas, se hace necesario un cambio en la relación entre docente y estudiante, el cual debe dejar de ser un trato de poder 
entre oprimido y opresor. Esta relación es visualizada por Freire (2006, p. 57) como una forma de violencia, ante la cual surgen siempre mecanismos de resistencia en torno a la búsqueda de una liberación donde, "mientras la violencia de los opresores hace de los oprimidos hombres a quienes se les prohíbe ser, las respuestas de éstos a la violencia de aquéllos se encuentra infundida del anhelo de búsqueda del derecho de ser". Dejar de lado esta relación de dominio implica, asimismo, el acabar con la función estática del docente, como agente dedicado únicamente al cumplimiento de contenidos, retomando la cordialidad, afabilidad y el respeto como elementos de motivación.

Igualmente, acabar con la opresión en las aulas significa el establecimiento de un trato horizontal, desde la humildad, donde los y las docentes, partiendo de la reflexión, se reconozcan como iguales a sus estudiantes. Esto conlleva poner fin a sentimientos de superioridad y tendencias excluyentes, que implican muchas veces situaciones de humillación, estereotipos y discriminaciones hacia las personas con las que se trabaja, sin tomar en cuenta el impacto emocional que acarrean estas prácticas en la configuración personal del estudiante. La sensibilización docente lleva también a una concientización acerca de la realidad en que se trabaja, sin dejar pasar situaciones adversas presentes en el plano educativo y social. Se debe formar parte de la cotidianeidad para impulsar la resolución de conflictos y promover la participación de los y las estudiantes en la disminución de tendencias de individualismo y discriminación que se transforman en situaciones de violencia. Esto, sobre todo, considerando la influencia del papel docente como ejemplo y objeto de estudio para sus estudiantes. Así, sus acciones determinarán buena parte de la toma de decisiones de los y las estudiantes con respecto a las problemáticas presentes en su entorno.

La sensibilidad y afectividad dentro del proceso educativo deben enfocarse en resaltar la identidad particular de cada uno de los y las estudiantes, reconociéndoles su dignidad como sujetos de derecho. Las diferentes realidades que se presentan en el entorno de aula, por lo tanto, no deben representar un obstáculo para una labor educativa integral, sino más bien una fortaleza, en virtud de establecer espacios para la reflexión desde diversas perspectivas. A la vez, la práctica reflexiva debe tener como finalidad el devolver la palabra a los y las estudiantes con el propósito de darles un lugar determinante en la configuración del conocimiento como seres pensantes. Al respecto, Gómez, D’ Antoni y otros 
(2013, p. 35), señalan el valor fundamental de la devolución de la palabra a los sujetos como contracara a la pedagogía tradicional, que otorga la voz únicamente a los educadores. Con ello se pretende que los sujetos reflexionen desde sus particularidades. Al tiempo que puedan llevar a cabo procesos de diálogo y alternativas para la resolución desde criterios éticos, siempre con la intencionalidad de generar la autoliberación de los educandos. Sobre la misma línea, Freire (2004, p. 98), insiste en la necesidad de romper barreras en la relación de diálogo entre docente y estudiante, propiciando la libertad de éste como sujeto crítico.

La sensibilidad, afectividad y reflexión en la labor docente se constituyen a partir de la buena práctica que va de la mano con el amor hacia lo que se hace y hacia los sujetos con que se trabaja. Este amor debe estar reflejado en la creación de cambios en el día con día; donde los y las educadores(as) no establezcan una práctica reiterativa centrada en las mismas dinámicas de clase, sino que, partiendo de las diferentes realidades de sus estudiantes, pueda ofrecer alternativas que sean atractivas, constructivas y motivadoras.

\section{Conclusión}

En el Sistema Educativo Nacional, la figura de docentes comprometidos(as) con un sentido de sensibilidad hacia su entorno de trabajo, sigue siendo una carencia, pues el modelo de educador(a) imperante en los Centros Educativos sigue respondiendo, en algunos casos, al docente lejano, sin un vínculo estrecho con los actores y actrices del proceso educativo. No obstante, se debe tener claro que la sensibilización, afectividad y reflexión no son saberes aprehendidos, sino que son cualidades adquiridas a través de la praxis, siendo el entorno de aula el principal espacio formativo donde las experiencias y vivencias determinan el carácter profesional. La proximidad afectiva, por lo tanto, parte de una decisión en la cual el docente, dentro de un sentir humanista, valora e integra a sus estudiantes dentro del proceso de enseñanza y aprendizaje.

Así pues, la sensibilidad, afectividad y reflexión no serán logradas sino hasta que se ponga en marcha una revolución de las conciencias, donde los y las docentes comprendan la importancia de estas actitudes dentro de los procesos de aula. Además, este complejo de actitudes no surgen de la reproducción de una discursividad, sino que nacen desde el sentir propio y el valorar y apreciar la labor y los sujetos que forman parte de ella. 


\section{Referencias bibliográficas}

Consejo Superior de Educación de Costa Rica (2008). El centro educativo decalidad como eje de la educación costarricense. Recuperado dehttp://www.uned.ac.cr/ ece/images/documents/doc2011_yrivera/un_centro_educativo_de_calidad.pdf

D’ Antoni, M y Gómez, J. (2013). La escuela en cuestionamiento: diálogos sobre la resistencia escolar en procesos pedagógicos existentes. San José, Costa Rica: Editorial Arlekin.

Freire, P. (2004). Cartas a quien pretende enseñar. Buenos Aires, Argentina: Siglo XXI Ediciones.

Freire, P. (2006). Pedagogía del oprimido. Buenos Aires, Argentina: Siglo XXI Ediciones.

Freire, P. (2008). Pedagogía de la autonomía: saberes necesarios para la práctica educativa. Buenos Aires, Argentina: Siglo XXI Ediciones.

Garcez, M. (2008). Osando decir la propia historia. San José, Costa Rica: Editorial SEBILA. 